\title{
On Minimum Variance Unbiased Estimator of the Parameter in $\mathrm{S}(\mathrm{d})$ Distribution
}

\author{
Nanjundan $\mathrm{G}^{1^{*}}$ and Suresh $\mathrm{R}^{2}$ \\ ${ }^{1}$ Department of Statistics, Bangalore University, Bangalore 560 056, India \\ ${ }^{2}$ Department of Statistics, Karnatak University, Dharwad 580 003, India \\ Email: nanzundan@gmail.com, sureshramaiah@gmail.com
}

\begin{abstract}
A new class of discrete distributions analogous to Burr family has been chararacterized by Sreehari (2010)[1]. The d-th member of this class is structurally equivalent to Poisson distribution. Nanjundan and Naika $(2012,2015)$ [2] [3] have discussed the maximum likelihood and the moment estimators of the parameter in the distribution. In this paper, a minimum variance unbiased estimator of the parameter is obtained and its properties are discussed. Further, an asymptotic comparison of these three estimators is done.
\end{abstract}

Keywords: Discrete distribution, maximum likelihood estimator, moment estimator, mimimum variance unbiased estimator, CAN, ARE.

\section{Introduction}

New distributions arise due to theoretical developments and situations wherein the existing statistical models or distributions become inadequate. Characterization of distributions is another source of getting new distributions. Discretization of continuous distributions also has added new entries to literature [See Chakraborty (2015)][4]. Estimation of parameters involved in such distributions is a worthy exercise.

The d-th member of the class of distributions characterized by Sreehari (2010)[1] has the probability mass function (pmf)

$$
p(x)= \begin{cases}(x+1-\theta) \frac{\theta^{x}}{(x+1) !}, & x=0,1,2, \ldots, 0<\theta<1 \\ 0 & , \text { otherwise. }\end{cases}
$$

The mean and the variance of this distribution are respectively $e^{\theta}-1$ and $e^{\theta}\left(2 \theta-e^{\theta}+1\right)$. This distribution being underdispersed is structurally equivalent to Poisson but does not belong to exponential family. Nanjundan and Naika (2012)[2] have named this as S(d) distribution. Sreehari (2010)[1] has illustrated that the $\mathrm{S}(\mathrm{d})$ distribution fits better than the Poisson distribution for a real life clinical trial dataset.

\section{Maximum Likelihood and Moment Estimators}

The maximum likelihood and the method of moment estimators of $\theta$ in the above pmf specified by (1) have been discussed by Nanjundan and Naika $(2012,2015)[2][3]$.

If $X=\left(X_{1}, X_{2}, \ldots, X_{n}\right)$ is a random sample on $\mathrm{X}$ having the pmf in (1), then the maximum likelihood estimate of $\theta$ is the solution of $\sum_{j=1}^{n} \frac{\theta}{x_{j}+1-\theta}-n \bar{x}=0$ where $\bar{x}=\frac{\sum_{j=1}^{n} x_{j}}{n}$. The maximum likelihood estimate (MLE) of $\theta$ does not have a closed form expression. Hence a numerical procedure like Newton-Raphson method can be employed to compute $\hat{\theta}_{m l e}$, the MLE of $\theta$.

By proving that the pmf in (1) satisfies the Cramer regularity conditions, Nanjundan and Naika (2012, 2015)[2][3] claim the following result which states the consistent asymptotic normality (CAN) of $\hat{\theta}_{m l e}$ :

$$
\sqrt{n}\left(\hat{\theta}_{m l e}-\theta\right) \stackrel{L}{\rightarrow} N\left(0, \frac{1}{I(\theta)}\right), \text { as } n \rightarrow \infty
$$


where $I(\theta)=-E\left[\frac{d^{2} \log p}{d \theta^{2}}\right]=\sum_{x=0}^{\infty} \frac{1}{x+1-\theta} \frac{\theta^{x}}{(x+1) !}+\frac{\left(e^{\theta}-1\right)}{\theta^{2}}$ is the Fisher information.

The infinite series $\sum_{x=0}^{\infty} \frac{1}{x+1-\theta} \frac{\theta^{x}}{(x+1) !}$ is not tractable but it is convergent for $0<\theta<1$ and it can be evaluated numerically correct to a desired number of decimal places.

The method of moment estimator (MME) of $\theta$ is $\hat{\theta}_{m m e}=\log (\bar{X}+1)$. The following result which states that the moment estimator is also consistent and asymptotically normal (CAN) is due to Nanjundan and Naika $(2012,2015)[2][3]$.

If $\underline{X}=\left(X_{1}, X_{2}, \ldots, X_{n}\right)$ is a random sample on $\mathrm{X}$ having the pmf specified in (1), then

$$
\sqrt{n}\left(\hat{\theta}_{m m e}-\theta\right) \stackrel{L}{\rightarrow} N\left(0, e^{\theta}\left(2 \theta-e^{\theta}+1\right) \frac{1}{(1+\theta)^{2}}\right) \text {, as } n \rightarrow \infty .
$$

The asymptotic efficiency of the MLE and the MME are compared with respect to the minimum variance unbiased estimator (MVUE) in Section-4.

\section{Minimum Variance Unbiased Estimator}

We now show that the pmf in (1) is complete. For a function $h($.$) , suppose that E_{\theta}[h(X)]=0$.

Then $\sum_{x=0}^{\infty} h(x)(x+1-\theta) \frac{\theta^{x}}{(x+1) !}=0 \Rightarrow \sum_{x=0}^{\infty} h(x)\left\{\frac{\theta^{x}}{x !}-\frac{\theta^{x+1}}{(x+1) !}\right\}=0$.

That is $h(0)(1-\theta)+h(1)\left(\frac{\theta}{1 !}-\frac{\theta^{2}}{2 !}\right)+h(2)\left(\frac{\theta^{2}}{2 !}-\frac{\theta^{3}}{3 !}\right)+\cdots=0$.

It is easily verifiable that $\left(\frac{\theta^{x}}{x !}-\frac{\theta^{x+1}}{(x+1) !}\right)>0, \forall x \geq 0$. Hence the coefficients of $h(0), h(1), h(2), \ldots$ are unequal and positive. In turn, we get $h(0)=h(1)=h(2)=\cdots=0$ and $h(x) \equiv 0$. Therefore $p(x, \theta), 0<\theta<1$ is a complete family. Though $p(x, \theta), 0<\theta<1$ is complete, since it does not belong to the exponential family, a minimum sufficient statistic cannot be identified in a straight forward manner. Also, the entire sample $\left(X_{1}, X_{2}, \ldots, X_{n}\right)$ is the only sufficient statistic.

When $\underline{X}=\left(X_{1}, X_{2}, \ldots, X_{n}\right)$ is a random sample on $X$ having the pmf mentioned in (1), define $Y_{j}=\left\{\begin{array}{l}0, \text { if } X_{j}=0 \\ 1, \text { if } X_{j} \neq 0 .\end{array}\right.$ Then $E\left(Y_{j}\right)=P\left(X_{j} \neq 0\right)=\theta, \forall j$. Take $T(\underline{X})=\frac{\sum_{j=1}^{n} Y_{j}}{n}$. Then $E(T(\underline{X}))=$ $\theta, \forall \theta \in(0,1)$ and hence $T(\underline{X})$ is an unbiased estimator of $\theta$.

It can be observed that, $P\left(X_{j} \neq 0\right)=\theta$ irrespective of the magnitude of $X_{j}$ and $P\left(X_{j}=0\right)=1-\theta, \forall j$. This is intrinistic due to the structure of the pmf. Hence the expected value of the proportion of nonzero $X_{j}^{\prime} s$ turns out to be an unbiased estimator of $\theta$.

Note that $Y_{j} \sim b(1, \theta)$ and $\sum_{j=1}^{n} Y_{j} \sim b(n, \theta)$. Since $-\infty<E\left(Y_{j}\right)<\infty$, by Kintchine's weak law of large numbers, $T(\underline{X}) \stackrel{P}{\rightarrow} \theta$, as $n \rightarrow \infty$. (Of course, $T(\underline{X}) \stackrel{a . s .}{\longrightarrow} \theta$, as $n \rightarrow \infty$ by Kolmogrov's strong law of large numbers.) That is $T(\underline{X})$ is consistent for $\theta$. Further, since $Y_{j}^{\prime} s$ are independent and identically distributed random variables with $E\left(Y_{j}\right)=\theta$ and $\operatorname{Var}\left(Y_{j}\right)=\theta(1-\theta)<\infty$, by Levy-Lindeberg central limit theorem

$$
Z_{n}=\frac{\sqrt{n}(T(\underline{X})-\theta)}{\sqrt{\theta(1-\theta)}} \stackrel{L}{\rightarrow} Z \sim N(0,1), \text { as } n \rightarrow \infty
$$

Therefore $T(\underline{X})$ is CAN for $\theta$.

Since $Y_{j} \sim b(1, \theta)$ and $b(1, \theta)$ is a member of the exponential family, $\sum_{j=1}^{n} Y_{j}$ is minimal sufficient for $\theta$. It is easy to see that $\operatorname{Var}(T(\underline{X}))=\frac{\theta(1-\theta)}{n}$ and the Fisher information of $T(\underline{X})$ is $\frac{n}{\theta(1-\theta)}$. Hence the Cramer-Rao lower bound for $\operatorname{Var}(T(\underline{X}))$ turns out to be $\frac{\theta(1-\theta)}{n}$. Therefore $T(\underline{X})$ is a minimum variance unbiased estimator (MVUE) for $\theta$ [See Bickel and Doksum (2001)][5]. 


\section{Asymptotic Relative Efficiency}

Using the asymptotic relative efficiency (ARE), an asymptotic comparison of these three estimators is done in this section.

The asymptotic relative efficiency of the proposed MVUE over the MLE is given by

$$
A R E_{M V U E v s M L E}=\frac{\text { Asymptotic variance of } \hat{\theta}_{\text {mle }}}{\text { Asymptotic variance of } \hat{\theta}_{\text {mvue }}} .
$$

Similarly, the asymptotic relative efficiency of the MVUE over the MME is

$$
A R E_{M V U E v s M M E}=\frac{\text { Asymptotic variance of } \hat{\theta}_{m m e}}{\text { Asymptotic variance of } \hat{\theta}_{\text {mvue }}} .
$$

The results are given in the table below.

Table 1. ARE of MVUE over MLE and MME of the parameter

\begin{tabular}{ccc}
\hline$\theta$ & Over MLE & Over MME \\
\hline 0.1 & 1.051 & 0.9624 \\
0.2 & 1.1055 & 0.9468 \\
0.3 & 1.1666 & 0.9514 \\
0.4 & 1.2409 & 0.9773 \\
0.5 & 1.3401 & 1.0296 \\
0.6 & 1.4885 & 1.1207 \\
0.7 & 1.7419 & 1.2816 \\
0.8 & 2.2658 & 1.6076 \\
0.9 & 3.885 & 2.5769 \\
\hline
\end{tabular}

It is evident from the table above that the MVUE is asymptotically more efficient than the MLE uniformly over the entire range of $\theta$. Whereas the MVUE is asymptotically more efficient than the MME when $\theta$ lies between 0.5 and 1.0.

\section{Simulation Study}

As a part of the further study of the performance of the three estimators, samples each of size $n=1000$ were generated from the $\mathrm{S}(\mathrm{d})$ distribution fixing $\theta=0.2,0.6$, and 0.8 and the $\mathrm{S}(\mathrm{d})$ distribution was fitted using the three estimators. The values of the Chisquare statistics for goodness of fit test have been calcualated and are presented in the following tables.

It is observed that the values of Chi-square goodness of fit statistics when the MVUE is used as the estimate of $\theta$ is lower than the corresponding values with respect to both the MME and the MLE for the chosen values of $\theta$. 
Table 2. Chisquare statistic values for $\theta=0.2$

\begin{tabular}{ccccc}
\hline $\mathrm{x}$ & Frequency & \multicolumn{3}{c}{ Expected Frequency } \\
\hline \hline & & MME & MLE & MVUE \\
\hline 0 & 802 & 808.5535 & 808.0808 & 802.0000 \\
1 & 185 & 173.1206 & 173.5027 & 178.3980 \\
2 & 13 & 17.1564 & 17.2383 & 18.3083 \\
\hline & Chi-square value & 1.875226 & 1.849699 & 1.783391 \\
\hline
\end{tabular}

Table 3. Chi-square statistic values for $\theta=0.6$

\begin{tabular}{ccccc}
\hline \multirow{x}{x}{} & Frequency & \multicolumn{3}{c}{ Expected Frequency } \\
\hline \hline \multirow{2}{*}{0} & 374 & 379.9613 & 378.2759 & 374.0000 \\
1 & 444 & 427.8147 & 428.4537 & 430.0620 \\
2 & 142 & 152.4952 & 153.2168 & 155.0523 \\
3 & 29 & 33.5704 & 33.8281 & 34.4871 \\
4 & 11 & 5.3947 & 5.4515 & 5.5975 \\
\hline & \multirow{2}{*}{ Chi-square value } & 7.874654 & 7.770024 & 7.637757 \\
\hline
\end{tabular}

Table 4. Chi-square statistic values for $\theta=0.8$

\begin{tabular}{ccccc}
\hline \multirow{x}{*}{$\mathrm{x}$} & Frequency & \multicolumn{3}{c}{ Expected Frequency } \\
\hline \hline & & MME & MLE & MVUE \\
\hline 0 & 197 & 197.9984 & 197.2116 & 197.0000 \\
1 & 477 & 480.3983 & 480.5538 & 480.5955 \\
2 & 237 & 235.6278 & 236.0059 & 236.1076 \\
3 & 77 & 68.7373 & 68.9229 & 68.9728 \\
4 & 12 & 14.4731 & 14.5273 & 14.5419 \\
\hline & \multirow{2}{*}{ Chi-square value } & 1.452886 & 1.41692 & 1.408795 \\
\hline
\end{tabular}

\section{Discussions and Summary}

The MVUE is asymptotically more efficient than the MLE of $\theta$. The MVUE is easy to compute for a given sample from the $\mathrm{S}(\mathrm{d})$ distribution whereas the MLE needs computing facilities. Further, since the MVUE is also CAN, it can be used for further inference on $\theta$. The simulations and computations in this paper have been done using the $\mathrm{R}$ software. 
Acknowledgments. The authors are very grateful to the referees whose suggestions greatly improved the presentation of the paper.

\section{References}

1. M. Sreehari, "On a class of discrete distributions analogous to burr family," Journal of Indian Statistical Association, 2010.

2. G. Nanjundan and T. R. Naika, "Estimation of parameter in a new discrete distribution analogous to burr family," in Proceedings of the International Conference on Information Technology and Computer Application Engineering. Lin, Sung, and Yao (Eds.), Taylor and Francis, London, 2014, pp. 399-402.

3. - "An asymptotic comparison of maximum likelihood and moment estimators of parameter in a new discrete distribution analogous to a burr distribution," ProbStat Forum, vol. 8, October, 2015.

4. S. Chakraborty, "Generating discrete analogues of continuous probability distributions: A survey of methods and constructions," Journal of Statistical Distributions and Applications, vol. 2, no. 6, 2015.

5. P. J. Bickel and K. A. Doksum, Mathematical Statistics. Prentice Hall, 2001. 